\title{
Editorial
}

Theme: Advances in Topical Delivery of Drugs

Guest Editor: S. Narasimha Murthy

\section{Approaches for Delivery of Drugs Topically}

\author{
S. Narasimha Murthy ${ }^{1,2}$
}

Received 15 November 2019; accepted 16 November 2019; published online 19 December 2019

The word topical dosage forms encompass dosage forms applied on the surface of the skin, nail, mucous membranes, and eye. Topical dosage forms are being preferred over other dosage forms as they provide local therapeutic effect when applied on the skin or mucous membranes (1). All these biological barriers are formidable and resist penetration of most of the drug molecules except a few which possess desirable physicochemical characteristics favoring penetration. Therefore, approaches to enhance drug delivery across biological barriers using passive and active transport is of interest to researchers to develop novel modalities of topical treatment of skin diseases and systemic disorders.

In the case of dermal delivery, stratum corneum, the outermost layer of the skin is tightly packed with keratinfilled cells, and the intercellular space is filled with saturated lipids, cholesterol, and fatty acids (2). The stratum corneum acts as a barrier to hydrophilic drugs and macromolecules. On the other hand, lipophilic drugs can penetrate the skin through partitioning into the intercellular lipids and via the transcellular route. Human skin comprises of hair follicles and sweat glands. In the case of topical delivery systems, percutaneous absorption of drug molecules is pivotal as the drug must be absorbed to an optimum concentration to maintain its therapeutic levels throughout their use (3). Drugs that are extremely hydrophilic or lipophilic would be poorly permeable across the skin. Drugs that are soluble in both aqueous and lipid phases only would be able to penetrate the skin (4).

In order to assist the passage of drug molecules through the stratum corneum, various transdermal permeation enhancers have been explored extensively. The chemicals that enhance skin permeability include various terpenes, pyrrolidones, fatty acids, fatty alcohols, surfactants, alcohols, glycols, and urea (5). Chemical permeation enhancers have

Guest Editor: S. Narasimha Murthy

\footnotetext{
${ }^{1}$ The University of Mississippi, University, Mississippi 8677, USA.

${ }^{2}$ To whom correspondence should be addressed. (e-mail: murthygroup@gmail.com)
}

been used to enhance the permeability of poorly permeable molecules through the biological barriers to (6). The chemical penetration enhancers may act via disruption of the lipids present in the stratum corneum, interaction with intercellular proteins, or via an improved partitioning of the drug into the stratum corneum. But chemical permeation enhancers have limited efficiency in enhancing the delivery of molecules which are required in huge doses to elicit pharmacological activity. They are also poorly efficient in enhancing the permeability of the barriers to macromolecules. Hence, exploring chemicals that can safely enhance permeability of a drug is still an interesting area of transdermal drug delivery research.

When macromolecules and biologicals need to be delivered into the skin, physical permeation enhancement techniques have been used. For example, iontophoresis across the cornea, skin, and nail has enhanced the delivery of therapeutic molecules that are required in large doses (transdermal delivery of sumatriptan for the treatment of migraine) and larger therapeutic molecules (hormones, peptide therapeutic agents). Treatment of skin disorders such as skin cancer, psoriasis, and dermatitis would be beneficial by using iontophoretic delivery of drugs (7). Likewise, researchers have investigated the use of magnetic field as a potential tool for delivery of molecules, called magnetophoresis. This technique applies magnetic field as an external driving force that enhances drug delivery across the skin (8). Another physical penetration enhancement technique is electroporation which involves the application of short, high voltage pulses to the skin. The electroporation technique forms small pores in the barrier leading to the delivery of larger molecules. Larger macromolecules including vaccines, insulin, oligonucleotides, and microparticles have been delivered using electroporation technique (9). For the last two decades, microneedle technique has been extensively investigated for transdermal delivery. It has been one of the most promising methods of delivery of drugs across the skin and mucous membrane (10). These microneedles overcome the top layer barrier of biological membranes and deliver the therapeutic agents close to the vicinity of the blood vessels located in the deeper layers. Using microneedles for transdermal delivery is minimally invasive, patient compliant, simple to use, and has huge commercial viability. 
Transdermal delivery of drugs is well recognized despite its limitations. Transdermal delivery allows for a controlled delivery with minimal side effects, increased efficiency, and maintenance of a therapeutic dose throughout topical administration. The objective of this issue is to focus on advances in the topical delivery of drugs using various approaches and evaluation of topical dosage forms for their quality and performance attributes.

\section{REFERENCES}

1. Garg T, Rath G, Goyal AK. Comprehensive review on additives of topical dosage forms for drug delivery. Drug Deliv. 2015;22(8):969-87. https://doi.org/10.3109/ 10717544.2013 .879355

2. Sahle FF, Gebre-Mariam T, Dobner B, Wohlrab J, Neubert RHH. Skin diseases associated with the depletion of stratum corneum lipids and stratum corneum lipid substitution therapy. Skin Pharmacol Physiol. 2015;28(1):42-55. https://doi.org/ 10.1159/000360009.

3. Dragicevic N, Maibach HI, Central E. Percutaneous penetration enhancers: chemical methods in penetration enhancement. Berlin: Springer; 2015.
4. Paudel KS, Milewski M, Swadley CL, Brogden NK, Ghosh P, Stinchcomb AL. Challenges and opportunities in dermal/ transdermal delivery. Ther Deliv. 2010;1(1):109-31. https:// doi.org/10.4155/tde.10.16.

5. Pathan IB, Setty CM. Chemical penetration enhancers for transdermal drug delivery systems. Trop J Pharm Res. 2009;8(2):173-9. https://doi.org/10.4314/tjpr.v8i2.44527.

6. Benson HAE. Transdermal drug delivery: penetration enhancement techniques. Curr Drug Deliv. 2005;2(1):23-33. https:// doi.org/10.2174/1567201052772915.

7. Mathur V, Satrawala Y, Rajput MS. Physical and chemical penetration enhancers in transdermal drug delivery system. Asian J Pharm. 2010;4(3):173-83. https://doi.org/10.4103/09738398.72115.

8. Murthy SN, Sammeta SM, Bowers C. Magnetophoresis for enhancing transdermal drug delivery: mechanistic studies and patch design. J Control Release. 2010;148(2):197-203. https:// doi.org/10.1016/j.jconrel.2010.08.015.

9. Denet A, Vanbever R, Préat V. Skin electroporation for transdermal and topical delivery. Adv Drug Deliv Rev. 2004;56(5):659-74. https://doi.org/10.1016/j.addr.2003.10.027.

10. Mitragotri S, Langer R, Prausnitz MR. Current status and future potential of transdermal drug delivery. Nat Rev Drug Discov. 2004;3(2):115-24. https://doi.org/10.1038/nrd1304.

Publisher's Note Springer Nature remains neutral with regard to jurisdictional claims in published maps and institutional affiliations. 